\title{
La teoría de conjuntos y la teoría del juego en el marco de las migraciones
}

Set Theory and Game Theory within the Migration Framework

\section{Defina a teoria ea teoria dos jogos no contexto da migração}

\section{Luis Fernando Sánchez Jaramillo* • Colombia}

Recibido el 18 de abril de 2011 - aprobado el 10 de mayo de 2011

\section{Resumen}

Objetivos. Con fundamento en la teoría de conjuntos y la teoría del juego, se examinan las dinámicas territoriales: primero, desde la apropiación del espacio y la significación que le otorgan las sociedades; segundo, a partir de la regulación de los espacios, con miras al aprovechamiento humano, la convivencia armónica y la construcción de fronteras como espacios de alteridad. Metodología. Se examina el problema de las migraciones a partir de la teoría de conjuntos y la teoría del juego para analizar las estrategias y las consecuencias de las movilidades de los grupos de migrantes. Resultados. A partir de ese examen se observó cómo la dinámica social, enfrentada o no con la naturaleza, se convierte en un juego en el que concurren diversos poderes que generan, al tiempo, conflictos con los cuales se dan movilidades sociales hacia otros campos de juego, otros territorios, en donde su dinámica tiene otras reglas que no siempre respetan las identidades y las tradiciones de las sociedades minoritarias tradicionales o de las nuevas minorías. Conclusiones. Los análisis de caso de los expertos y tomando como elemento propositivo de análisis la teoría de conjuntos y la teoría de juegos, se logra observar la manera cómo se asientan las comunidades humanas luego de efectuar los continuos desplazamientos. Existe acuerdo en que las relaciones territoriales son dinámicas, que las fronteras se modifican constantemente, que las movilidades son un fenómeno que ha caracterizado la historia de la humanidad, y es posible recurrir a elementos como las teorías mencionadas para observar y analizar mejor estas dinámicas.

Palabras claves: territorio, medio ambiente, fronteras, migraciones.

* Licenciado en Ciencias Sociales•Magister en Filosofía • Universidad de Caldas•luissanchezj@gmail.com 


\begin{abstract}
Objectives. This paper aims to analyze territory dynamics based on the set theory and game theory from three different perspectives. Firstly, it is seen from the appropriation of space and the meaning given by the societies; secondly, from the regulation of space oriented to the human benefit and from the regulation of good living practices within the territory, and thirdly, from the building of frontiers as a space of the otherness. Methodology. It examines the problem of migrations from the set theory and game theory perspective to analyze strategies and consequences of mobilities of migrant groups. Results. Based on this analysis, it is realized that the social dynamics, with or against nature, becomes a game in which various powers meet. These powers generate, in turn, conflicts where social mobilities take place towards other game fields, other territories. There dynamics have other rules that not always respect their identities and the traditions of minor societies or those of the new societies. Conclusions. The analyses of case reports/studies by experts based on the set theory and game theory perspective show the way how human communities settle after continuous displacements. There exists an agreement on the fact that territorial relations are dynamic, that borders are permanently modified, and that mobilities have been a fact all along human history. It is then possible to recur to elements of the abovementioned theories to better observe and analyze this dynamics.
\end{abstract}

Keywords: Territory, environment, frontiers, migrations.

\title{
Resumo
}

Objetivos. Com fundamento na teoria de conjuntos e a teoria do jogo, examinam se as dinâmicas territoriais: desde a apropriação do espaço e a significação que lhe outorgam as sociedades; a partir da regulação dos espaços, com miras ao aproveitamento humano, da convivência harmônica e a construção de fronteiras como espaços de alteridade. Metodologia. Examina se o problema das migrações a partir da teoria dos conjuntos e a teoria do jogo para analisar as estratégias e as conseqüências das mobilidades dos grupos de migrantes. Resultados. A partir desse exame observou se como a dinâmica social, enfrentada ou não com a natureza, converte se num jogo no que concorrem diversos poderes que geram, ao tempo, conflitos com os quais se dão mobilidades sociais dirigidas a outros campos de jogo, outros territórios, onde sua dinâmica tem outras regras que não sempre respeitam as identidades e as tradições das sociedades minoritárias tradicionais ou das novas minorias. Conclusões. Os analises dos expertos e tomando como elemento propositivo de analises a teoria de conjuntos e a teoria de jogos, logra se observar a maneira como se assentam as comunidades humanas logo de efetuar os contínuos deslocamentos. Existem acordos em que as relacionem territoriais são dinâmicas, que as fronteiras modificam constantemente, que as mobilidades são um fenômeno que tem caracterizado a historia da humanidade, e é possível recorrer a elementos como as teorias mencionadas para observar e analisar melhor estas dinâmicas.

124 Palavras chave: Território, meio ambiente, fronteiras, migrações. 


\section{Introducción}

\section{Nuevas migraciones y movilidades... nuevos territorios}

El Grupo de Investigación Territorialidades de la Universidad de Caldas se conformó para reflexionar alrededor de la concepción de los estudios territoriales, a partir de la antropología, la sociología y la historia; en ellos se busca analizar, interpretar, explicar y reconstruir las diferentes posturas y prácticas socioculturales que las distintas sociedades tienen sobre y desde el territorio, entendido éste como una reordenación del espacio cuyos signos culturales caracterizan a una sociedad ${ }^{1}$. Las reflexiones sobre territorio y territorialización fueron puestas en común en los célebres Seminarios Internacionales sobre Territorio y Cultura ${ }^{2}$, el primero realizado en octubre de 1999 y el último en noviembre de 2010, de los cuales se hace necesario destacar la quinta versión, que se desarrolló en conjunto con los doctores María Teresa Rodríguez del Centro de Investigaciones y Estudios Superiores en Antropología Social CIESAS y Manuel Uribe del Instituto Nacional de Antropología e Historia INAH. En el marco de este V Seminario Internacional sobre Territorio y Cultura, celebrado en la veracruzana ciudad de Xalapa en México en septiembre de 2006, se examinaron las teorías y métodos en el estudio de las transformaciones territoriales, la migración y la movilidad poblacional; se trató de una reflexión teórica aplicada a estudios de caso o etnografías que apuntaron a dar contexto y debate amplio en la relación entre los temas. (Nates \& Uribe, 2007: pp. 7-14).

Cuestionamientos como ¿Cuál es el rol -función y lugar- de los Estados-nación actuales en los procesos que relacionan territorialidades y migración? ¿Cómo se expresa la relación entre migración (o movilidad) y procesos de configuración territorial? ¿Cómo y desde dónde las transformaciones territoriales, la migración y la movilidad poblacional son generadores de nuevas identidades individuales y colectivas? ¿Cómo la migración y la movilidad de población originan tipos nuevos de identidades emergentes generando una suerte de categorización de grupos sociales, tanto en los lugares de partida como en los de llegada? ¿Cómo y desde dónde, en la medida en la que estos grupos sociales se recomponen y se integran (comportamientos, economía, socialización, manejo espacial, cultural, etcétera), se reorganizan implícitamente? ¿Cómo, qué y desde dónde las migraciones y las movilidades de población aportan tanto a quienes se quedan como a quienes les reciben?, son algunos de los que se intentaron responder para demostrar que las realidades contemporáneas, sin contraponer lo local a lo global, tratan de pensar el mundo en términos diferenciales al relacionar los conceptos de globalización, territorio, migración y movilidad de población, evidenciando que los

1 El Grupo de Investigación Territorialidades de la Universidad de Caldas fue fundado en 1998.

2 Este Seminario lo organiza el Grupo de Investigación Territorialidades de la Universidad de Caldas desde 1999. 
Estados no dominan su territorio tanto como administran sus condiciones globales, esto es, los marcos legislativos que permiten la apertura a los capitales financieros, la inversión de las multinacionales, la mediatización de la información en forma de libre circulación de bienes y servicios, etcétera; por lo tanto, se hace necesario revisar la actualidad y el alcance de los conceptos de globalización, mundialización, innovación, integración así como su significado en relación con la estrategia de recomposición de los Estados nacionales, con miras a los análisis culturales, sociopolíticos y económicos de transformaciones territoriales, las migraciones y la movilidad poblacional.

En ese orden de ideas, trece expertos ${ }^{3}$ se aproximaron a develar estas inquietudes:

(...) a partir del examen del fenómeno de la organización territorial de los Estados mediante un análisis crítico de reformas constitucionales y nuevas constituciones aparecidas entre 1995 y el 2005; el cuestionamiento sobre la pertinencia que tiene que referirse al final de las sociedades tradicionales a partir del estudio de los regionalismos y las identidades latinoamericanas; la dualidad de las políticas de identidad europea mediante las lecciones conceptuales de dos estudios de caso; los órdenes significativos del mundo y los procesos migratorios en contextos de globalización; un enfoque interdisciplinario para el estudio del territorio indígena en los Altos de Chiapas; la sistemogénesis de una aristocracia campesina de caficultores; la migración indígena, patrones sociales y resignificación cultural entre los indígenas Sikuani del Medio río Guaviare en Colombia entre 1958 y el 2001; las fronteras de las visiones del mundo y de las identidades territoriales; el papel de las fronteras en la conformación de la nación cubana; el ordenamiento territorial como una alternativa de encuentro para diversas lógicas; las rupturas, compromisos, anhelos, retornos y que desengañasen las relaciones espacio temporales con el pueblo de origen por parte de los emigrados; reflexiones desde las migraciones forzadas en Colombia, y la migración analizada desde los puntos de partida. (Nates \& Uribe, et al.).

Una mirada transversal de estos asuntos, que con apoyo en la Teoría de Conjuntos y la Teoría del Juego se exponen más abajo, es examinada aquí explorando los puntos en común y las distancias que tomaron los panelistas en torno a la problemática de estudio prevista en el Seminario.

\section{Metodología}

\section{Teoría de conjuntos}

Es inevitable cruzar por las miradas que los expertos, que concurrieron al Seminario de Xalapa, le dieron a sus trabajos sin abordar una postura histórico-filosófica; primero, porque indudablemente las relaciones sociales se dan en los territorios en una

3 Sus nombres y los de sus ponencias aparecen registrados en la bibliografía que acompaña este artículo, que como se dijo, sirve de base al planteamiento que intento construir. 


\section{Gráfica comprensiva de los movimientos migratorios y de las territorialidades}

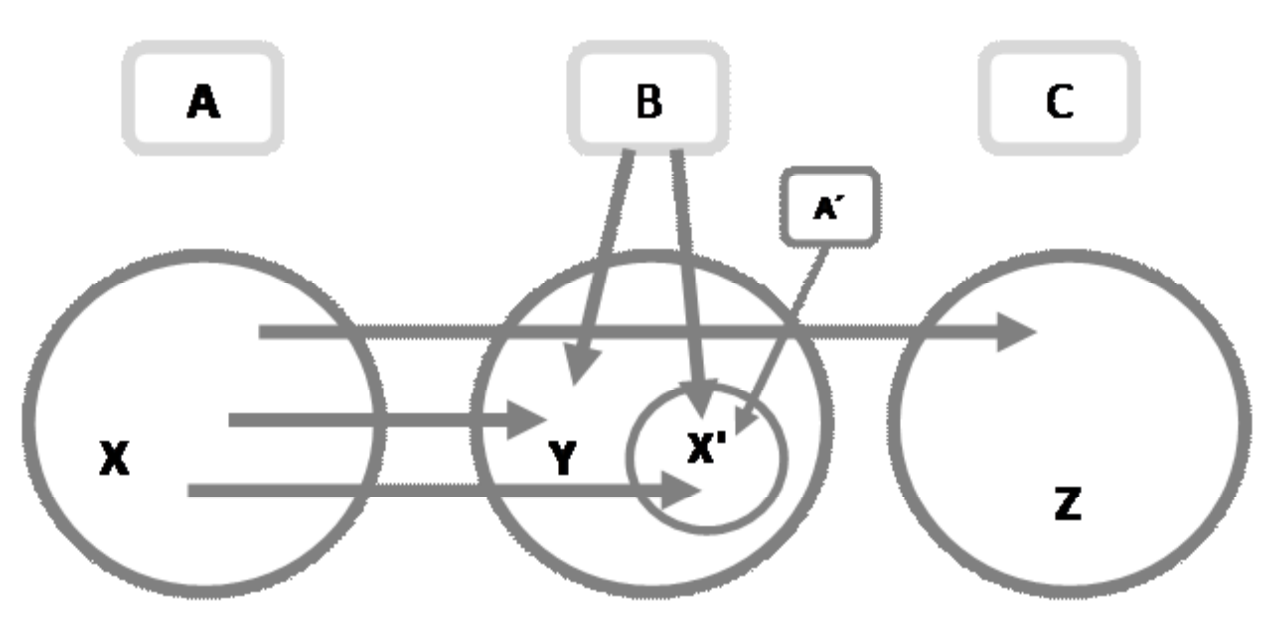

Fuente: elaboración propia

dimensión histórica que, al tiempo, se mueve en las coordenadas espacio-tiempo y, segundo, por el apoyo tangencial en la teoría de $\operatorname{conjuntos}^{4}$ y en la denominada teoría del juego. Para examinar las dinámicas de los grupos sociales en la conformación de territorios, es necesario describir la manera como ellas pueden funcionar.

Sea entonces un espacio $\mathbf{A}$ ocupado por una población $\mathbf{X}$, ese espacio es territorializado cuando en él se dan una serie de relaciones políticas, sociales, culturales (educativas, religiosas, deportivas), económicas, etcétera, que llevan a esa población a significar de tal manera ese espacio, que se convierte en un territorio sobre el cual la población tiene una relación de identidad. Y sea un espacio $\mathbf{B}$ ocupado por una población $\mathbf{Y}$, territorializado de manera parecida que $\mathbf{A}$. Si aceptamos que en ambos territorios se dan relaciones endógenas entre los miembros que lo comparten y con el espacio mismo, también es posible aceptar que pueden existir relaciones exógenas, esto es, entre los miembros del espacio $\mathbf{A}$ con los de $\mathbf{B}$ mediante múltiples modalidades; por ejemplo, de un miembro de $\mathbf{A}$ que se interesa en un aspecto de $\mathbf{B}$ y al contrario, o todos los miembros de $\mathbf{A}$ que se interesan en particularidades de $\mathbf{B}$ o viceversa.

Los intereses mutuos, particulares o comunales, pueden generar una suerte de categorías ${ }^{5}$ que se distinguen según el grado de arraigo que se tenga en el territorio

$4 \quad$ Llama la atención la propuesta teórico-metodológica de María Teresa Ayllón Trujillo quien, para explicar lo que ella llama el continuum migración-arraigo, se fundamenta en la Teoría General de Sistemas. Cfr., Ayllón, 2006: pp. 207-225.

5 Algunas de esas categorías son: Movilidades, migraciones, territorio, territorialidades, desplazamientos, fronteras, identidades, etcétera. 
con el que cada quien se identifica; un miembro de $\mathbf{A}$ puede tener un interés temporal para conocer el espacio $\mathbf{B}$ o el territorio de $\mathbf{B}$ si su intensión final es regresar para permanecer en $\mathbf{A}$ o seguir hasta $\mathbf{C}$; pero también un miembro de $\mathbf{A}$ puede preferir o ser obligado a permanecer indefinidamente en $\mathbf{B}$. En el primer caso si existiera algo en $\mathbf{B}$ que lo atrajera tanto como para cambiar de lugar de vivienda; por ejemplo, estudiar, trabajar, etcétera; y en el segundo caso, si existiera en $\mathbf{A}$ alguna situación que lo obligue a abandonarlo sin que $\mathbf{B}$ sea un destino voluntariamente buscado.

Podrá encontrarse el caso de la población $\mathbf{X}$ que se encuentre motivada para ir a $\mathbf{B}$, pero es también probable que sea obligada a ir a $\mathbf{B}$; por ejemplo, en caso de guerra en A. En contraste, en $\mathbf{B}$ se aceptaría acoger a algún miembro de la población $\mathbf{X}$ que se encuentre en $\mathbf{A}$ para que visite $\mathbf{B}$ y se integre con la población $\mathbf{Y}$, pero muy poco dispuesta a tolerar a algún miembro de $\mathbf{X}$ para permanecer indefinidamente en $\mathbf{B}$ y menos para interactuar constantemente con Y. Otra posibilidad consiste en que una parte de la población $\mathbf{X}$, que antes se encontraba en $\mathbf{A}$, llegue a ocupar un subespacio $\mathbf{A}^{\mathbf{\prime}}$ en $\mathbf{B}$ formando una población $\mathbf{X}$ ', ese espacio se significaría hasta alcanzar una identidad parecida a la que originalmente $\mathbf{X}^{\prime}$ tenía en $\mathbf{A}$.

No obstante la simpleza de la formalización que explican los conceptos de Movilidad, Migración y Territorio, habrá unas nuevas categorías que emergen de esta red de relaciones y que se complejizan en tanto las relaciones pasen a otro nivel suprarelacional. Cuando un miembro de $\mathbf{X}$ en $\mathbf{A}$ pasa temporalmente a $\mathbf{B}$ para interactuar con $\mathbf{Y}$ evoca a $\mathbf{A}$ debido al significado que para $\mathbf{X}^{\prime}$ tiene $\mathbf{A}$, esto es, que $\mathbf{X}^{\prime}$ se identifica con $\mathbf{A}$, y que guarda la esperanza real de regresar alguna vez a $\mathbf{A}$ para interactuar con $\mathbf{X}$.

Pero si las razones para que $\mathbf{X}$ permanezca en $\mathbf{B}$ obedecen a factores lejos de su control y se le impidiera relacionarse con miembros de $\mathbf{Y}$ en $\mathbf{B}$, por razones de orden jurídico o cultural o económico, entonces $\mathbf{X}$ ' buscaría a otros miembros $\mathbf{X}$ de $\mathbf{A}$ en $\mathbf{B}$ que se encontraran en una situación como la suya e intentaría conformar un subespacio $\mathbf{A}^{\prime}$ dentro de $\mathbf{B}$ con el fin de formar una colonia que se resignificaría como una forma de evocar $\mathbf{A}$. Esa misma colonia de $\mathbf{A}$ en $\mathbf{B}$, es decir A' se habría desarraigado de $\mathbf{A}$, se habría desterritorializado para territorializar $\mathbf{B}$ a través de $\mathbf{A}^{\prime}$.

Tanto en $\mathbf{A}$ como en $\mathbf{B}$ las relaciones entre $\mathbf{X}$ como entre $\mathbf{Y}$ están matizadas por una normatividad expresa o tácita, dependiendo de la participación de sus miembros en el diseño de esa normatividad o de los micro poderes que sientan mandatos que se transmiten por tradición cultural, cuando no por imposición del más fuerte. A y B pueden tener un tipo de normatividad que los relacione mediante tratados de cooperación o tratados tácitos impuestos por la fuerza de la costumbre; sin embargo, el conflicto se puede formar cuando se legisla en $\mathbf{B}$ para su población sin tomar en cuenta 128 a la población $\mathbf{X}$ en $\mathbf{A}^{\prime}$. 


\section{Teoría del juego}

La teoría del juego ${ }^{6}$ se ocupa sobre todo de examinar lo que ocurre cuando los hombres se relacionan de forma racional; esto implica abordar el problema de las relaciones estratégicas y se basa en la aceptación tácita o explícita de unas reglas de juego. Esta teoría se aplica generalmente en la economía pero también en la ciencia política en lo que llamaríamos el juego del poder. El modelo que permite explicar los fenómenos sociales en relación con la naturaleza se fundamenta, no sólo en la presentación descriptiva que nos posibilita la teoría de conjuntos, para hacer un análisis multivariado de esas relaciones, sino que, además, una teoría del juego permite explicar otros aspectos de las relaciones sociales con el Estado.

Para explicar mejor este aspecto se puede imaginar un juego cualquiera en el que participan los niños; afirmar o emplear la palabra juego y a los niños como actores supondría un aspecto de poca seriedad en la construcción del model; sin embargo, no hay nada más serio que el juego de los niños. Para que haya un juego es necesario que los participantes identifiquen un objeto de juego, es decir, qué vamos a jugar; posteriormente se acuerdan las reglas del juego, esto es, las normas que regirán la acción en la que los jugadores participaran. Nótese que antes de cualquier juego de niños hay un momento en el que ellos discuten esas normas y posteriormente si el desarrollo del juego lo exige proponen modificarlos y establecen nuevos acuerdos.

Puesto que los juegos son tan variados, tan novedosos y tan ingeniosos como es capaz de concebir la mente humana, quizás se piense que el juego de solitario exija mayor responsabilidad que el juego colectivo, pues sólo la autoconfrontación daría cuenta del autoengaño. Sin embargo, un juego colectivo pasa por la confrontación colectiva y, a menos que se respeten las normas o se convenga su modificación, podrían surgir conflictos que también el juego debería resolver sobre la base de las normas y los acuerdos o, podrían generar un juego abusivo que tenga como resultado, a la postre, el fin del juego; por ejemplo, en un juego de fútbol, el objeto es introducir el balón en el arco contrario el mayor número de veces posible, evitando al mismo tiempo que lo haga el equipo contrario; las reglas, por populares, son generalmente conocidas y los instrumentos, entre otros, el campo de fútbol y el balón. Si el balón fuera un instrumento de propiedad de uno de los jugadores, es decir, no es un bien común, es muy probable que su dueño quiera imponer algunas normas que le convenga o que decida llevarse el balón al no poder imponerlas y, en consecuencia, dar por terminado el juego.

Otro tipo de juego es aquel en el que se emplean las fichas, por ejemplo, el ajedrez ${ }^{7}$, este juego cumple con los requerimientos mencionados: un objeto y unas reglas de jue-

6 La Teoría de juegos fue creada por Von Neumann Morgenstern en su libro The Theory of Games Behavior publicado en 1944. No obstante, existen numerosas referencias de cómo la teoría del juego ha tenido diferentes usos en las explicaciones filosóficas.

7 En realidad el juego del ajedrez, llamado por sus conocedores como juego ciencia, tiene amparadas sus fichas por reglas mundialmente conocidas que le otorgan a cada tipo un movimiento específico en una situación dada. Lo importante aquí es que esas fichas no pueden cambiar de rol ya que par- 
go. A este respecto los jugadores juegan en concordancia con las reglas, las fichas son jugadas y, por tanto, ignoran que lo son, lo que implica estar sometidas a eventuales cambios de reglas sin importar su punto de vista, el de las fichas, si es que fuera posible que la manifestaran.

Una aplicación de la teoría del juego en el territorio o en la sociedad, el resultado sería más o menos el siguiente: todo hombre es miembro de una sociedad en tanto se relacione con otros hombres para compartir diversos intereses culturales y aprovechar los recursos que provee la naturaleza independiente de la forma como se dé ese aprovechamiento. El espacio es el campo del juego que aquí se llamará el juego social, las reglas están representadas en la constitución y demás cuerpo de leyes aprobadas por el poder legislativo y puestas en funcionamiento por el poder judicial, y los jugadores son cada miembro de la sociedad con sujeción a las citadas normas; no obstante, si bien el cuerpo legislativo y los cuerpos ejecutivo y judicial son nombrados directamente o indirectamente por la sociedad, ya que ellas son sus representantes, existe en la escala de juegos el juego por el poder que puede distorsionar el objeto que busca el juego social, por ejemplo las tiranías, los autoritarismos.

Cuando el juego por el poder está por encima del juego social, los miembros de esa sociedad pueden tener dos comportamientos: el primero, es que algunos miembros de la sociedad, por ejemplo los académicos, tengan conciencia del juego y, en consecuencia, acuerden modificar las reglas del juego para no perder de vista el objeto del juego social, y el segundo, es que otros miembros de la sociedad carezcan de esa conciencia y se conviertan en fichas que son jugadas a favor del juego por el poder. Sólo cuando existe conciencia del juego es posible participar en su modificación si se hace necesario, y jugar activamente para alcanzar el propósito del juego social, lo contrario es estar al vaivén de invisibles jugadores que tienen otros propósitos generalmente diferentes a los de la mayoría.

\section{Instrumentos del juego, la naturaleza y la sociedad}

La naturaleza suele expresarse de manera imprevista a pesar de que las disciplinas que la estudian han alcanzado mayores niveles para comprenderla; sin embargo, la humanidad sigue sin entender la inseparable relación que tiene con esa naturaleza. El estudio de la interacción que ha existido entre el hombre y la naturaleza, que las Ciencias Sociales han denominado Territorio, viene permitiendo la comprensión mediante la reflexión de los cambios que suceden entre esos dos elementos y de las interacciones de los complejos que identificamos como relaciones sociales y medio natural. Si bien territorio es un concepto polisémico empleado indistintamente por varias disciplinas,

ticipan inconscientemente en el juego, pero imaginemos qué lo controlaría y cómo se modificaría si las fichas tuvieran autonomía para moverse por fuera de las reglas que conocemos. Si bien es cierto que existen versiones informatizadas de este juego, los computadores juegan con fundamento en las reglas conocidas por todos. 
hay ya un consenso entre los científicos para reconocer que no se trata de un depósito de los productos naturales y artificiales creados por el hombre, sino que el territorio se constituye sólo cuando el hombre se apropia del espacio y establece allí unas relaciones socio-espaciales, para el aprovechamiento de los recursos naturales, generando todo tipo de conflictos que dejan toda una huella cultural producto de las identidades que los miembros de la sociedad logran construir. (Llanos, 2006: pp. 81-88).

En efecto, los denominados desastres naturales habrá que leerlos, no sólo en función de la dinámica de la propia naturaleza, sino a partir de la relación que ella ha tenido con la sociedad a la que ha albergado, es decir, una lectura construida sobre los efectos que esos desastres naturales causan a los miembros de esas sociedades. En el primer caso, la historia ha dado cuenta suficiente del continuo aprovechamiento de los recursos naturales; no se aprendió suficientemente de los ancestros el respeto por la naturaleza, el tránsito de las sociedades agrarias a las sociedades industrializadas dieron paso a un aprovechamiento indiscriminado que se conoció con el adjetivo explotación, que ha causado el creciente desequilibrio natural del que han hablado los ecologistas y cuyas evidencias ahora son palpables. Y el segundo, el de las relaciones de la naturaleza con la sociedad.

\section{Reglas de juego: las constituciones, el ordenamiento territorial y las políticas de diversidad cultural}

Ya se ha dicho que la Constitución es la norma por excelencia de cada Estado, es la base sobre la que se resuelven los conflictos de intereses de todos los sectores de la sociedad, se determina la organización administrativa y se reglamenta toda la relación entre la población y el poder en un democrático equilibrio para unos y para otros. Las constituciones han sido fruto de muchos debates, disputas y enfrentamientos armados y son las conquistas más estimadas de los pueblos, las constituciones amparan los derechos y deberes de los naturales y extranjeros en relación con su territorio, tal como dice López (2006): "en ella la organización territorial es un espacio reglado delimitado territorial y políticamente”. Sin embargo, parte de la normativa que guía al territorio es el denominado ordenamiento territorial, cuyo propósito es guiar los destinos territoriales para buscar un desarrollo equilibrado. La tradición también muestra que en el área rural el desarrollo territorial se ha asumido como "un proceso de transformación productiva e institucional en un espacio determinado cuyo fin es reducir la pobreza rural". Por lo tanto, el Ordenamiento Territorial debe orientarse a "fortalecer la concertación y la interacción entre agentes sociales y territorios, y capitalizar la voluntad política de quienes toman las decisiones para la colectividad" (Velásquez, 2006, pp. 165-172).

En este sentido, las normas generales que guían a las sociedades o las específicas que intentan el desarrollo territorial, algunas veces no alcanzan a abarcar todos los intereses sociales. Las migraciones como forma de movilidad significan cómo las territorialida- 
des son perdidas, readquiridas; es evidente que en algunos países, especialmente los europeos, el idioma sumado a la territorialidad genera una mayor identidad territorial; de este modo, surgen de la tríada de grupos autóctonos, las minorías de inmigrantes y las denominadas minorías históricas, una suerte de etnogénesis con la que, además, se generan un sinnúmero de retos por la diversidad etnocultural que se da en las organizaciones subregionales y supraregionales que caracterizan a las sociedades de hoy (Dietz, 2006, pp. 45-66). De acuerdo con lo anterior, se enfrentan en las comunidades territorializadas unas comunidades corporativas no territorializadas que obligan a los gobiernos a diseñar y aplicar políticas que, a decir de Dietz (2006), son de doble rasero, es decir, desarrollo de políticas de identidad absurdas que intentan distinguir entre una etnogénesis nacional o regional basada en el territorio y otra que carece de él como los inmigrantes.

Si bien el fenómeno de la inmigración se refiere a un grupo conformado por sujetos que ingresan a un espacio no territorializado originalmente por ellos, gracias a la permisividad o no de la normatividad o reglas de juego establecidas por el poder legislativo en ejercicio del juego político, ese ingreso se realiza de diversas formas: de manera legítima, es decir, en observancia de todas las reglas del juego, o a la sombra de esas reglas, lo que da lugar a las autoridades que administran el juego para que, aplicando las mismas reglas, excluyan del juego a quienes infringen la ley, por ejemplo, la de migraciones. Sin embargo, la mayoría de las reglas del juego se adaptan a los jugadores y fichas nativas, vale decir, a los detentadores del poder y a los miembros de la sociedad, que son presa inconsciente del poder de los primeros, pero poco a los inmigrantes legales.

No obstante las reglas de juego expresadas en las constituciones para los inmigrantes, otro tipo de normas, consignadas en la tradición cultural, dificultan el disfrute de derechos legales en territorios ajenos, obligando a los inmigrantes a practicar juegos de supervivencia, a través de ordenamientos territoriales no formales, que a la postre obligan a los administradores del juego político a diseñar políticas para el manejo de la diversidad cultural.

\section{Resultados}

\section{El Juego en acción: causa y prácticas de migración}

Algunos aspectos que se destacan en las relaciones territoriales es el tema de las fronteras que son, para Hernández (2006), un constructo de la mente humana; esto significa que el mundo actual es el producto de fronteras impuestas por la mente. Históricamente la disputa por los derechos ha modificado esas fronteras y ha generado todo tipo de movilidades entre ellas las que sufre Colombia como la migración forzada (Osorio, 2006, pp. 187-206); de algún modo la historia de la humanidad es la historia de la construcción y reconstrucción de fronteras y de des- 
territorialización y reterritorialización; sin embargo, las sociedades modernas han construido también unos modelos que surgen de la realización personal y que se acentúan por la seducción publicitaria que han generado los medios de comunicación y las nuevas tecnologías, lo cual ha empujado las sociedades hacia otras fronteras generando grandes migraciones internacionales principalmente como producto de la racionalidad liberal y, especialmente, de la idea de progreso. No obstante, la movilidad, en cualquiera de sus formas, genera una serie de relaciones entre territorio, identidad y acción colectiva, que cuando chocan con el desconocimiento de las condiciones socioculturales de los lugares de destino se entra en crisis y se vuelve utópica la idea de progreso que se ha dado a los países desarrollados (Hernández, 2006, pp. 67-80).

\section{Modificaciones del juego, modificaciones del territorio}

Una de las posibilidades que permiten el análisis del territorio desde una mirada multidisciplinar, con lo cual se logra un conocimiento más amplio del territorio, es la relación que existe entre tradición y progreso y la pérdida de la cultura y la identidad.

Es el caso del territorio indígena de los altos de Chiapas, estudiado por Llanos (2006: 81-88), que se ha modificado con los procesos de globalización; allí el ancestral cultivo del maíz que se asocia con la identidad cultural indígena fue reemplazado por el cultivo de la flor que se convirtió en un importante elemento económico, ha transformado el territorio indígena y la concepción de tiempo con paradigma de progreso, lo cual concuerda con algunos autores (Ariel, 2006. pp. 173-186) que manifiestan que las funciones sociales y productivas de las comunidades rurales y tradicionales que se vinculan al mercado capitalista atomizan dichas sociedades y declinan la asociatividad de la vida comunitaria; sin embargo, Ariel (2006) aclara, en el caso de los habitantes de la Esperanza en la Península de Yucatán, como ellos han demostrado el surgimiento de una especie de neoruralidad que ha desdibujado las fronteras físicas formales entre las regiones y las grandes urbes ya que las nuevas infraestructuras permiten mayor integración regional que antes, siempre y cuando la tecnología permita compartir su patrimonio cultural. Empero, otro tipo de ejemplos de dinámicas colonizadoras es el de los indígenas Sikuani del Guaviare colombiano (González Gómez, 2006, pp. 113-130) quienes a través de distintos juegos y formas de poder fueron ocupados y obligados a emigrar hasta encontrarse en medio de micro poderes del Estado y de la globalización; es el producto de las dinámicas nacionales y la configuración del Estado para la colonización de fronteras que obligaron a los Sikuani al sometimiento de la imposición de patrones ajenos a su cultura, que los obligaron a movilizarse para ocupar territorios cultural y ambientalmente distintos a los suyos. 


\section{Fronteras en el juego: entre identidad y tradición}

Como se sabe, la complejidad del concepto de territorio obliga a enfocarlo desde otras perspectivas como la del poder gubernamental, la administración jurídica o la del enfoque socio- antropológico, esto no es más que una demostración de la existencia de múltiples escalas y niveles que han sido históricamente constituidos. Uno es el caso de las fronteras interiores de Cuba que responden a un proceso histórico-económico y político que contrasta con fronteras exteriores que son de definición más compleja. (Venegas, 2006, pp. 115-164) y otro, el del territorio del Lago de Usina Hidroeléctrica (UHE) Serra da Mesa (Almeida, 2006, pp. 131-114) en donde se plantea la discusión de la relación que hay entre los espacios identitarios de una especie de cultura nativa frente a las reconfiguraciones identitarias a las que son sometidas las sociedades tradicionales ante las amenazas que genera este tipo de megaproyectos, generando al mismo tiempo lo que denominaríamos acciones simbólicas de frontera donde se construyen identidades como constructo histórico en una zona que se denomina lugar de alteridad, en donde se delimitan realidades estableciendo igualmente espacios de conflicto.

En América Latina, la denominada independencia del dominio español generó una concepción histórica decimonónica caracterizada por las ideas positivistas que se amparaban en la idea de progreso (Le Goff, 1995), pero al mismo tiempo por una idea de nacionalismo de acuerdo con la visión de aquella época. Las noveles naciones construyeron una visión histórica desde el centro hacia la periferia que se denominó historia nacional, una historia signada en medio de las confrontaciones por la escogencia del modelo de gobierno. En aquella época poco importaban las regiones en tanto el poder económico no tuviera algo que aportar pero al mismo tiempo se pensaba en una historia de punto final, una historia dogmática a la cual nada había que agregar y en la que las regiones y las localidades nada tenían que decir; la historia nacional que se construyó desde el centro no logró la identidad de algunas regiones.

Ahora, las celebraciones del segundo centenario de la independencia de los países de América Latina, han puesto de manifiesto la posibilidad de cambiar el paradigma regional en el que tengan peso las localidades y las regiones para construir una historia local y regional cuya sumatoria da como resultado la verdadera historia nacional. Esta historia es más cercana a los miembros de las sociedades que se integran al mundo mediante la identificación con su propio pasado y se identifican con el presente y con el futuro aportando a la solución de problemas que ahora son comprensibles para ellos. Partir del conocimiento de la historia local y regional no implica una regresión al nacionalismo, si no que es revalorizar las culturas de los pueblos que habitan las regiones, permitiendo aclarar su papel en un ambiente de globalización. (González Martínez, 2006, pp. 29-44) A pesar de las discusiones epistemológicas de las denomi134 nadas historia regional y microhistoria, (Miño, 2002,pp. 867-897) y entendiendo las 
discusiones de las escuelas ítalo-mexicanas sobre historia regional y microhistoria hay que reconocer el sentido pragmático que tiene una historia que se construye de la periferia al centro.

Ejemplos más concretos de identidad y tradición son el que genera el concepto de frontera y el papel de las migraciones de colonos en las aristocracias campesinas de caficultores (Tulet, 2006, pp. 89-112), en este caso el sentido de frontera de la colonización cafetera se entiende o como una delimitación entre dos espacios distintos o como un espacio en vía de organización. Entendiendo primero, que las formas de origen de los caficultores se dieron por iniciativa del Estado nacional, o por la acción de actores privados o por coyunturas del país. Tulet (2006) reconoce que en la primera forma el Estado nacional facilitó la venta de lotes provocando la llegada de inversionistas extranjeros y obligando a los campesinos a cultivar café en parte de sus tierras; esta dinámica facilitó el fomento de explotaciones familiares. Luego, los empresarios urbanos, que trataron de encontrar una actividad sustitutiva de otros cultivos, se conformaron en sociedades agrarias en donde el café se convierte en la base de la identidad de ese grupo por su carácter empresarial, con cuya prosperidad se alcanza la conquista y el mantenimiento del poder político. Y, finalmente, el relativo poder del café hace que el cafetero de una sociedad familiar reconozca que puede ser más pobre pero más independiente en contraste con las grandes haciendas que son las afectadas por la caída de los precios; los cafeteros familiares que sostienen la caficultura tradicional producen más que café sin perder el signo distintivo de identidad que existe con ese producto en esta parte del mundo, ya que el café es más que un producto, es una historia y unas dinámicas territoriales específicas, resultado que llevan a la caficultura a conformarse en territorios de identidad, de migración y de territorialidad.

\section{Conclusiones}

Con base en los análisis de caso de los expertos que concurrieron al Seminario de Xalapa en 2006, con el exclusivo fin de entender las similitudes del análisis territorial a partir de las movilidades y migraciones de diversas sociedades, y tomando como elemento propositivo de análisis la teoría de conjuntos y la teoría de juegos, se logra, en primer lugar, observar la manera cómo se asientan las comunidades humanas luego de efectuar los continuos desplazamientos. Existe acuerdo en que las relaciones territoriales son absolutamente dinámicas, que las fronteras se modifican constantemente, que las movilidades son un fenómeno que ha caracterizado la historia de la humanidad, pero es necesario recurrir a elementos como las teorías mencionadas para observar y analizar mejor estas dinámicas.

Se ha examinado cómo las sociedades establecen reglas de juego explícitas como las constituciones y las leyes en las que también se adoptan planes reordenamiento territorial, e implícitas como los pactos tácitos que llamamos costumbre o relaciones de 
tradición; no obstante, la importancia que tiene el análisis de las reglas de juego social consiste fundamentalmente en la inequidad que ellas conllevan hacia sociedades cuyo poder puede ser mayor, dependiendo de los factores sociopolíticos o económicos que le favorezcan, pero también en detrimento de otros miembros que se encuentran al margen de ese poder o de esos micro poderes.

Los grupos más desprovistos de las ventajas del poder se ven obligados, o a iniciar movimientos hacia otros lugares en los que puedan construir el suyo propio, o hacia donde puedan alcanzar una cierta realización económica, o donde se puedan sentir más seguros, no obstante llegar a otros territorios se corre el riesgo de inadaptación o de desprotección de la identidad cultural del sujeto que se moviliza, el cual se ve obligado, no sólo a construir un territorio en un espacio diferente al suyo con ayuda de los suyos, sino, tal vez, a regresar y reterritorializar el espacio que antes tenía y que ahora ofrece nuevas condiciones para subsistir.

Un aspecto especial, que es paradójico, lo constituye la movilización por razones de la naturaleza. Para algunos la naturaleza es y el hombre se encuentra ahí para comprenderla, especialmente sabiendo que forma parte de ella; pero no hay que olvidar que principalmente durante la segunda mitad del siglo XX la humanidad la ha agredido; los ecosistemas que dan equilibrio al paisaje natural han sido reemplazados por otros sistemas indiscriminados que han causado desequilibrios sin precedentes cuyas consecuencias vemos hoy. Desde tiempos de los griegos, Aristóteles había observado la complejidad de la naturaleza y la forma como ella atentaba en ocasiones contra el hombre; no obstante, cualquiera sea la causa que altere el curso normal de la naturaleza, oleadas de hombres han tenido que abandonar sus tierras temporal o permanentemente, a veces perdiendo sus pertenencias materiales cuando no las vidas de sus familiares; en este caso, parece más doloroso el desarraigo para llegar a tierras de nadie o a tierras ajenas en la que nadie los quiere. Los desórdenes naturales son menos llevaderos cuando ni siquiera es posible volver porque no quedan ni los recuerdos.

Tanto los empujones que produce la naturaleza, como los que producen los juegos de poder o la aspiración que conduce al paradigma del progreso, o el simple gusto de conocer, son causas de la movilidad. El destino no siempre garantiza que los nuevos lugares sean mejores ni peores, pero la acción social consciente ayuda a que se modifiquen las reglas de juego a favor de las minorías; es una forma de superar la imposición de las instituciones sobre las sociedades que las minorías tradicionales o las nuevas minorías tengan participación como una armónica sociedad natural.

Las migraciones, a la par que parten de un juego de poder, son también complejos que afectan los territorios de origen y de destino. Los países subdesarrollados pierden mano de obra joven que van a beneficiar la de los países desarrollados, al tiempo que muchos migrantes buscan en otros territorios las oportunidades que no tienen en su país de origen. Algunos países atraen migrantes extranjeros acomodados para que les 
dejen divisas; no obstante, queda una franja de sobrevivientes, vividores o delincuentes que juegan al margen de las reglas, de una parte, porque en el lugar de origen se dedicaban a poco o, de la otra, porque las condiciones halladas en el nuevo territorio no les favorecen y encuentran en el mundo subterráneo la trampa o la oportunidad de participar en juegos siniestros para ellos y para las sociedades.

No obstante, siempre se establecen fronteras que cercan el mundo y que seguirán modificandose para formar un campo de alteridad en el que cada conflicto se resuelva con nuevos acuerdos y nuevos juegos que respeten y compartan identidad y tradición.

\section{Referencias}

Almeida, M. G. (2006). "Fronteiras de visões de mundo e de identidades territoriais - o território plural do Norte Goiano-Brasil". Universidade Federal de Goiás, Brasil. En Nates \& Uribe, (Coordinadores). Nuevas Migraciones y Movilidades... Nuevos Territorios. Manizales-Colombia: Universidad de Caldas. pp. 131-114.

Ariel de Vidas, A. (2006). "Rupturas, compromisos, anhelos, retornos, desengaños... Las relaciones espacio-temporales con el pueblo de origen". Centro de Estudios Mexicanos y Centroamericanos (CEMCA), México. En Nates \& Uribe, (Coordinadores). Nuevas Migracionesy Movilidades...Nuevos Territorios. Manizales-Colombia: Universidad de Caldas. pp. 173-186.

Ayllón Trujillo, M. T. (2006). "El continuum migración-arraigo". Universidad San Luis Potosí, México. En Nates \& Uribe, (Coordinadores). Nuevas Migraciones y Movilidades...Nuevos Territorios. Manizales-Colombia: Universidad de Caldas. pp. 207-225.

Dietz, G. (2006). "La dualización de las políticas de identidad en Europa: Lecciones conceptuales a partir de dos estudios de caso (Andalucía y Flandes)" Universidad de Andalucía, España/ Universidad de Veracruz, México. En Nates \& Uribe, (Coordinadores). Nuevas Migraciones y Movilidades...Nuevos Territorios. Manizales-Colombia: Universidad de Caldas. pp. 45-66.

González Gómez, L. M. (2006). "Migración indígena, patrones sociales y resignificación cultural entre los indígenas Sikuani del Medio Río Guaviare, Colombia”, 1958-2001. Universidad Bolivariana- Universidad Nacional, Colombia. En Nates \& Uribe, (Coordinadores). Nuevas Migracionesy Movilidades...Nuevos Territorios. Manizales-Colombia: Universidad de Caldas. pp. 113-130.

González Martínez, J. R. (2006). "Regionalismos e identidades latinoamericanas ¿final de las sociedades tradicionales?” Instituto de investigaciones Histórico- sociales Universidad Veracruzana, México. En Nates \& Uribe, (Coordinadores). Nuevas Migraciones y Movilidades... Nuevos Territorios. Manizales-Colombia: Universidad de Caldas. pp. 29-44.

Hernández Pulgarín, G. (2006). "Ordenes significativos del mundo y procesos migratorios en contextos de globalización”. Grupo de Investigación Territorialidades, Universidad de Caldas, Colombia. En Nates \& Uribe, (Coordinadores). Nuevas Migraciones y Movilidades... Nuevos Territorios. Manizales-Colombia: Universidad de Caldas. pp. 67-80. 
Le Goff, J. (1995). Pensar la Historia. Barcelona: Ed. Altaya.

López Trigal, L. (2006). "La recomposición territorial de los Estados en las constituciones recientes". Universidad de León, España. En Nates \& Uribe, (Coordinadores). Nuevas Migraciones y Movilidades... Nuevos Territorios. Manizales-Colombia: Universidad de Caldas. (1528).

Llanos Hernández, L. (2006). "Un enfoque interdisciplinario para el estudio del territorio indígena en Los Altos de Chiapas", Universidad de Chapingo, México. En Nates \& Uribe, (Coordinadores). Nuevas Migraciones y Movilidades...Nuevos Territorios. Manizales-Colombia: Universidad de Caldas. pp. 81-88.

Miño Grijalva, M. (2002). “Existe la historia regional?”. En Historia de México Colegio de México pp.867-897.

Morgenstern, Von N. (1944). The Theory of Games Behavior.

Nates \& Uribe, (Coordinadores) (2007). Nuevas Migraciones y Movilidades... Nuevos Territorios. Manizales-Colombia: Universidad de Caldas. pp. 7-14.

Osorio Pérez, F. E. (2006). "Algunas reflexiones desde las migraciones forzadas en Colombia". Universidad Javeriana, Colombia. En Nates \& Uribe, (Coordinadores). Nuevas Migraciones y Movilidades...Nuevos Territorios. Manizales-Colombia: Universidad de Caldas. pp. 187-206.

Tulet, J. C. (2006). “Caficultores: sistemogénesis de una aristocracia campesina”. CNRS, Université de Toulouse Le Mirail, Francia. En Nates \& Uribe, (Coordinadores). Nuevas Migraciones y Movilidades... Nuevos Territorios. Manizales-Colombia: Universidad de Caldas. pp. 89-112.

Velásquez López, P. A. (2006). "El ordenamiento territorial... una alternativa de encuentro para diversas lógicas”. Grupo de Investigación Territorialidades IGAC-UPTC, Colombia. En Nates \& Uribe, (Coordinadores). Nuevas Migraciones y Movilidades... Nuevos Territorios. Manizales-Colombia: Universidad de Caldas. pp. 165-172.

Venegas Delgado, H. (2006). "Frontera y región en la conformación de la colonia en Cuba". Instituto de Historia de Cuba, Cuba. En Nates \& Uribe, (Coordinadores). Nuevas Migraciones y Movilidades...Nuevos Territorios. Manizales-Colombia: Universidad de Caldas. pp. 115-164. 